\title{
Rotation-Covariant Texture Learning Using Steerable Riesz Wavelets
}

\author{
Adrien Depeursinge, Antonio Foncubierta-Rodriguez, \\ Dimitri Van de Ville, Senior Member, IEEE, and Henning Müller
}

\begin{abstract}
We propose a texture learning approach that exploits local organizations of scales and directions. First, linear combinations of Riesz wavelets are learned using kernel support vector machines. The resulting texture signatures are modeling optimal class-wise discriminatory properties. The visualization of the obtained signatures allows verifying the visual relevance of the learned concepts. Second, the local orientations of the signatures are optimized to maximize their responses, which is carried out analytically and can still be expressed as a linear combination of the initial steerable Riesz templates. The global process is iteratively repeated to obtain final rotationcovariant texture signatures. Rapid convergence of class-wise signatures is observed, which demonstrates that the instances are projected into a feature space that leverages the local organizations of scales and directions. Experimental evaluation reveals average classification accuracies in the range of $97 \%$ to 98\% for the Outex_TC_00010, the Outex_TC_00012, and the Contrib_TC_00000 suites for even orders of the Riesz transform, and suggests high robustness to changes in images orientation and illumination. The proposed framework requires no arbitrary choices of scales and directions and is expected to perform well in a large range of computer vision applications.
\end{abstract}

Index Terms-Texture classification, feature learning, steerability, rotation-covariance, illumination-invariance, wavelet analysis.

\section{INTRODUCTION}

$\mathbf{L}$ OCAL organization of scale and direction is key for visual pattern discrimination [2]. This property has been fueling research activities in image processing

Manuscript received June 4, 2013; revised September 18, 2013 and November 25, 2013; accepted December 2, 2013. Date of publication December 20, 2013; date of current version January 13, 2014. This work was supported in part by the Swiss National Science Foundation under Grants 205320-141300/1, PBGEP2_142283 and PP00P2-146318, in part by the CIBM, and in part by the EU 7th Framework Program in the context of the Khresmoi Project under Grant FP7-257528. The associate editor coordinating the review of this manuscript and approving it for publication was Prof. Sina Farsiu.

A. Depeursinge is with the MedGIFT group, Business Information Systems, University of Applied Sciences Western Switzerland, Sierre 3960, Switzerland, the University Hospitals and University of Geneva, Geneva 1211, Switzerland, and also with the Department of Radiology, School of Medicine, Stanford University, CA 94305 USA (e-mail: adrien.depeursinge@hevs.ch).

A. Foncubierta-Rodriguez and H. Müller are with the MedGIFT group, Business Information Systems, University of Applied Sciences Western Switzerland, Sierre 3960, Switzerland, and also with the University Hospitals and University of Geneva, Geneva 1211, Switzerland (e-mail: antonio.foncubierta@hevs.ch).

D. Van de Ville is with the Ecole Polytechnique Fédérale de Lausanne, Lausanne 1015, Switzerland, and also with the University of Geneva, Geneva 1211, Switzerland (e-mail: dimitri.vandeville@epfl.ch).

Color versions of one or more of the figures in this paper are available online at http://ieeexplore.ieee.org.

Digital Object Identifier 10.1109/TIP.2013.2295755 and pattern recognition since their early days [5], [20], [23], [28], [53], [61]. Nevertheless, approaches from computer vision are still lacking to elegantly leverage this fundamental property. A proper characterization of the local organizations of scales and directions raises several challenges. First, the analysis of scale and direction information should not be carried out separately since the co-occurrences of several scales and various directions are often key discriminating properties. Second, accurate characterizations of the Nyquist domain (i.e., scale or spatial frequency) and the angular domain (i.e., directions) are required to catch subtle inter-pattern differences. Third, the notion of locality is of leading importance to avoid the drowning of relevant pattern properties. Two patterns that are visually very different can have identical global distributions of scales and directions. The multiresolution theory of the wavelet transform provides an elegant solution to the locality problem for scale characterization [35]. However, less convincing solutions are available for comprehensive local direction characterization. Separable wavelets are biased along the vertical and horizontal directions [56]. The majority of texture analysis approaches (e.g., gray-level co-occurrence matrices (GLCMs), run-length encoding (RLE), oriented filterbanks and wavelets (Gaussian, Gabor, Leung-Malik (LM), Maximum Response (MR) [6], [32], [42], [45], [60], non-separable wavelets), and histograms of oriented gradients (HOG) used in scale-invariant feature transform (SIFT) [34]) all require arbitrary choices of directions for image analysis. A common practice is to explore directions by uniformly sampling the angular domain with a typical step of $45^{\circ}$ to span 4 directions in 2-D. While providing a comprehensive characterization of the directions in a neighborhood of radius $r=1$, the amount of discarded pixels increases quadratically with $r$ as $(2 r+1)^{2}-8 r-1$. Local binary patterns (LBP) [39] yield local descriptions of multiple directions using the pixel sequence along perimeters of radius $r$. However, they do not come with a multiresolution framework and finding appropriate values of $r$ requires optimization. Several researchers extended wavelet theory to tackle this issue. Brushlets [36], curvelets [4], ridgelets [13] and contourlets [11], [41] were introduced as approaches for comprehensively partitioning the angular space for neighborhoods of any radius. An interesting alternative is to use steerable filterbanks, allowing a continuous characterization of the directions [15], [49]. Steerable filterbanks allow obtaining filter coefficients at any arbitrary local orientation from a linear 
combination of the basis filters; the linear weights can be determined analytically. It was used with wavelet filterbanks (e.g., the steerable pyramid [18], [50]) enabling multiscale and multi-orientation analysis with infinitesimal angular precision.

In order to recognize identical pattern instances that have different local orientations, rotation invariant or covariant features are required. Invariant features do not vary when the input is transformed, while covariant ones have a bilinear relationship with respect to a transformation of the input. A common practice to obtain rotation-invariance is to sum the outputs of the operators over all directions. Rotation-invariant convolutional operators can be obtained with isotropic functions (e.g., [58] or the Schmid (S) filter [47]). Unfortunately, invariant approaches entail the risk of missing important directional properties (i.e., local anisotropy). Rotation-covariant features allow keeping local directional information while normalizing the operators' outputs over the instances. Rotation-covariant LBPs are obtained by using "uniform" circular pixels sequences that are rotationinvariant [39]. Rotation-covariant SIFT (i.e., RIFT [31]) measures HOG orientations relatively to the local dominant gradient orientation. However, the extraction of both uniform sequences and HOG are exhaustive and do not specifically model discriminative patterns. They also require arbitrary choices of the radius of the circular neighborhoods. Rotation covariance using steerable filters has also been proposed [8], [12]. In previous work, we locally aligned the first template of steerable Riesz wavelets to obtain rotation-covariant texture features [8]. Such a template models $N$ th-order directional derivatives and has a strong angular selectivity. A limitation of this approach is that this template does not model the local organization of the directions as it only seeks the one prevailing.

Several researchers proposed to learn filters from data, aiming at modeling local organizations of scales and directions [9], [17], [40], [44], [46], [48], [54], but few of them are coupled with a rotation-covariant framework. In this work, we propose iterative rotation-covariant texture learning using steerable Riesz wavelets as an effective way of exploiting local organizations of scales and directions of visual patterns. In a first step, optimally discriminative texture signatures (i.e., in the sense of structural risk minimization [59]) are built from the data. $N$ th-order Riesz filterbanks constitute texture dictionaries, from which the richness and angular selectivity is controlled by the order $N$ of the transform. Optimal linear combinations of the multiscale Riesz templates are obtained using support vector machines (SVM) for a given one-versus-all (OVA) classification task, which does not make assumptions on scales and directions. Class-wise texture signatures are obtained, allowing for visual assessment of the learned texture patterns. In a second step, the orientations of the learned signatures are locally oriented to maximize their response, which can be obtained analytically as a linear combination of the initial coefficients. Starting from the coefficients of the locally oriented signatures, the whole procedure is repeated iteratively until convergence of the texture signatures.

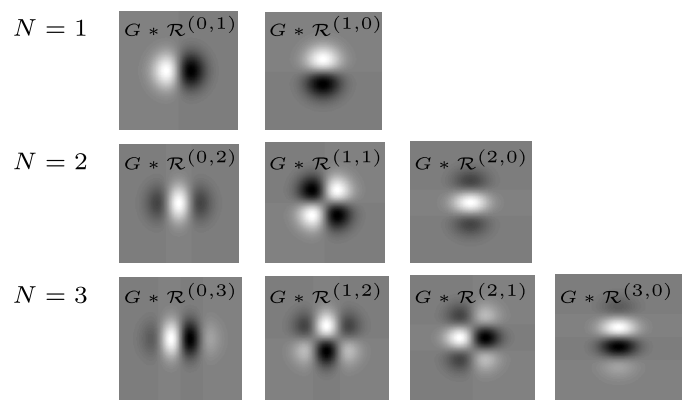

Fig. 1. Templates corresponding to the Riesz kernels convolved with a Gaussian smoother for $N=1,2,3$.

\section{Material AND Methods}

Our approach for iterative rotation-covariant texture learning using steerable Riesz wavelets is described in this section. The Riesz transform and associated filterbanks are explained in Section II-B. The iterative texture learning framework and the validation scheme used to evaluate it are described in Sections II-C-II-D and II-F-II-G respectively.

\section{A. Notations}

A generic $d$-dimensional signal $f$ indexed by the continuous-domain space variable $\boldsymbol{x}=\left(x_{1}, \ldots, x_{d}\right) \in \mathbb{R}^{d}$ is considered. The $d$-dimensional Fourier transform of $f$ is noted as:

$$
\begin{aligned}
f(\boldsymbol{x}) \stackrel{\mathcal{F}}{\longleftrightarrow} \hat{f}(\boldsymbol{\omega}) & =\int_{\mathbb{R}^{d}} f(\boldsymbol{x}) e^{-j\langle\boldsymbol{\omega}, \boldsymbol{x}\rangle} \mathrm{d} x_{1} \ldots \mathrm{d} x_{d}, \\
\text { with } \boldsymbol{\omega} & =\left(\omega_{1}, \ldots, \omega_{d}\right) \in \mathbb{R}^{d} .
\end{aligned}
$$

\section{B. Steerable Riesz Filterbanks}

The Riesz transform is a multidimensional extension of the Hilbert transform, which maps any function $f(x)$ to its harmonic conjugate and is a very powerful tool for mathematical manipulations of periodic signals [52]. For a 2-D signal $f(\boldsymbol{x})$, the $N+1$ components of the $N$ th-order Riesz transform $\mathcal{R}^{N}$ are defined as:

$$
\mathcal{R}^{N}\{f\}(\boldsymbol{x})=\left(\begin{array}{c}
\mathcal{R}^{(0, N)}\{f\}(\boldsymbol{x}) \\
\vdots \\
\mathcal{R}^{(n, N-n)}\{f\}(\boldsymbol{x}) \\
\vdots \\
\mathcal{R}^{(N, 0)}\{f\}(\boldsymbol{x})
\end{array}\right),
$$

with $n=0,1, \ldots, N$. A singular kernel $\mathcal{R}^{(n, N-n)}\{f\}(\boldsymbol{x})$ is defined in the Fourier domain as:

$$
\mathcal{R}^{(n, N-n)}\{f\}(\boldsymbol{x}) \stackrel{\mathcal{F}}{\longleftrightarrow} \mathcal{R}^{(n, \widehat{N-n)}}\{f\}(\boldsymbol{\omega}),
$$

where

$$
\begin{aligned}
\mathcal{R}^{(\widehat{n, N-n)}}\{f\}(\boldsymbol{\omega})= & \sqrt{\frac{N}{n !(N-n) !}} \\
& \frac{\left(-j \omega_{1}\right)^{n}\left(-j \omega_{2}\right)^{N-n}}{\|\boldsymbol{\omega}\|^{N}} \hat{f}(\boldsymbol{\omega}),
\end{aligned}
$$


with $\omega_{1,2}$ corresponding to the frequencies along the two image axes $x_{1,2}$. The multiplication with $j \omega_{1,2}$ in the numerator corresponds to partial derivatives of $f$ and the division by the norm of $\boldsymbol{\omega}$ in the denominator makes that only phase information is retained. Therefore, $\boldsymbol{\mathcal { R }}^{N}$ yields allpass ${ }^{1}$ filterbanks with directional (singular) kernels $\mathcal{R}^{(n, N-n)}$ [56]. The Riesz transform commutes with translation, scaling or rotation. The orientation of the Riesz components is determined by the partial derivatives in Eq. (2). The first-order Riesz transform corresponds to a phase-only gradient. The higher-order versions as specified in (2) are obtained by regrouping the $2^{N}$ Riesz filters into $N+1$ components by commutativity of convolution (e.g., $\partial^{2} / \partial x \partial y$ is equivalent to $\left.\partial^{2} / \partial y \partial x\right)$. The Riesz kernels $\mathcal{R}^{(n, N-n)}$ convolved with Gaussian kernels for $N=1,2,3$ are depicted in Fig. 1.

The Riesz filterbanks are steerable [15], [56], which means that the local response of each component $\mathcal{R}^{(n, N-n)}$ of an image $f(\boldsymbol{x})$ rotated by an arbitrary angle $\theta$ can be derived analytically from a linear combination of the responses of all components of the filterbank using a steering matrix $\boldsymbol{A}^{\theta}$ as:

$$
\mathcal{R}^{N}\left\{f^{\theta}\right\}(\mathbf{0})=\boldsymbol{A}^{\theta} \mathcal{R}^{N}\{f\}(\mathbf{0}) .
$$

The derivation of $\boldsymbol{A}^{\theta}$ is made explicit in Appendix A. Multiscale versions of these filterbanks are obtained by coupling the Riesz transform with Simoncelli's multi-resolution framework based on isotropic band-limited wavelets [57].

\section{Texture Signature Learning}

Qualitatively, the first Riesz component of even order corresponds to a ridge profile whereas for odd ones we obtain an edge profile, but much richer profiles can be obtained by linear combinations of the different components [9].

In order to optimally exploit the richness of the feature detectors encompassed in the Riesz components for a given pattern classification task, an appropriate weighting scheme of the energies of the responses of the Riesz components $E\left(\mathcal{R}^{(n, N-n)}\{f\}(\boldsymbol{x})\right)$ is required. The goal is to build an optimal texture signature $\Gamma_{c}^{N}$ of the class $c$ (versus all) from a linear combination of the Riesz components as:

$$
\begin{aligned}
\Gamma_{c}^{N} & =\boldsymbol{w}^{\mathrm{T}} \mathcal{R}^{N} \\
& =w_{1} \mathcal{R}^{(0, N)}+w_{2} \mathcal{R}^{(1, N-1)}+\cdots+w_{N+1} \mathcal{R}^{(N, 0)},
\end{aligned}
$$

where $\boldsymbol{w}$ contains the weights of the respective Riesz components. A multiscale texture signature is obtained as an extension of Eq. (4) with multiscale Riesz filterbanks [9] as:

$$
\begin{aligned}
\Gamma_{c}^{N}= & w_{1}\left(\mathcal{R}^{(0, N)}\right)_{s_{1}}+w_{2}\left(\mathcal{R}^{(1, N-1)}\right)_{s_{1}}+\cdots \\
& +w_{J(N+1)}\left(\mathcal{R}^{(N, 0)}\right)_{s_{J}},
\end{aligned}
$$

where $s_{j}, j=1, \ldots, J$ is the scale index. Scale-wise signatures $\Gamma_{c, j}^{N}$ can be obtained when using only weights and corresponding Riesz templates at the scale $j$. An example of the construction of a texture signature $\Gamma_{c}^{8}$ for a given texture of class $c$, order $N=8$ and scale $j=1$ is illustrated in Fig. 2.

\footnotetext{
${ }^{1}$ Except for the DC component.
}

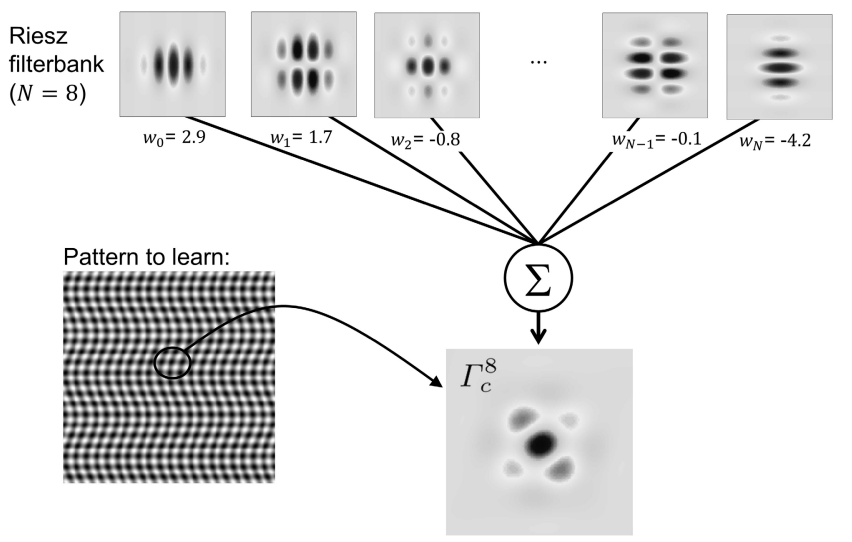

Fig. 2. Example of the construction of a texture signature $\Gamma_{c}^{8}$ using a linear combination of the Riesz templates $\mathcal{R}^{(n, N-n)} . \Gamma_{c}^{8}$ is visually similar to the essential "stitch" of the pattern.

Then $l_{2}$-norm support vector machines (SVM) are used to find the optimal weights $\boldsymbol{w}^{\mathrm{T}}=\left(w_{1} \ldots w_{N+1}\right)$ in the sense of structural risk minimization [59], [22] as:

$$
\begin{aligned}
& \min _{\tilde{\boldsymbol{w}}, \xi, b}\left\{\frac{\|\tilde{\boldsymbol{w}}\|^{2}}{2}+C \sum_{i=1}^{m} \xi_{i}\right\} \quad \text { subject to } \\
& y_{i}\left(\tilde{\boldsymbol{w}}^{\mathrm{T}} \phi\left(\boldsymbol{x}_{i}\right)-b\right) \geq 1-\xi_{i}, \quad \xi_{i} \geq 0,
\end{aligned}
$$

where $\xi_{i}$ are the slack variables of the soft margin, $C$ is the cost of the errors, $\boldsymbol{x}_{i}$ are the pattern instances $i=1, \ldots, m$ expressed in terms of the energies of the Riesz components with $y_{i}$ being their corresponding labels, and $\phi\left(\boldsymbol{x}_{i}\right)$ a function that maps each instances to a higher dimensional space. The following dual problem equivalence is used to solve Eq. (6):

$$
\begin{array}{r}
\min _{\boldsymbol{\alpha}}\left\{\frac{1}{2}+\boldsymbol{\alpha}^{\mathrm{T}} Q \boldsymbol{\alpha}-\boldsymbol{e}^{\mathrm{T}} \boldsymbol{\alpha}\right\} \quad \text { subject to } \\
\boldsymbol{y}^{\mathrm{T}} \boldsymbol{\alpha}=0, \quad 0 \leq \alpha_{i} \leq C,
\end{array}
$$

where $\boldsymbol{e}=[1, \ldots, 1]^{\mathrm{T}}$ is a vector of ones, $Q$ is an $m \times m$ positive semidefinite matrix as $Q_{i, j}=y_{i} y_{j} K\left(\boldsymbol{x}_{i}, \boldsymbol{x}_{j}\right)$, and $K\left(\boldsymbol{x}_{i}, \boldsymbol{x}_{j}\right)=\phi\left(\boldsymbol{x}_{i}\right)^{\mathrm{T}} \phi\left(\boldsymbol{x}_{j}\right)$ can be any positive definite kernel function. The primal-dual relationship yields the weights $\tilde{\boldsymbol{w}}$ from the support vectors in the high-dimensional kernel space:

$$
\tilde{\boldsymbol{w}}=\sum_{i=1}^{m} y_{i} \alpha_{i} \phi\left(\boldsymbol{x}_{i}\right)
$$

However, $\tilde{\boldsymbol{w}}$ cannot be used in Eqs. (4) or (5), because it is in the higher dimensional space determined by the mapping function $\phi(\boldsymbol{x})$ of the kernel. Therefore, the expression of weights $\boldsymbol{w}$ in the initial feature space is obtained from support vectors (i.e., $\alpha_{i}>0$ in Eq. (8)) as:

$$
\boldsymbol{w}=\sum_{i=1}^{m} y_{i} \alpha_{i} \boldsymbol{x}_{i}
$$

A graphical representation of the expression of $\boldsymbol{w}$ in the initial space of the Riesz component is shown in Fig. 3. The contribution of each Riesz component is determined 


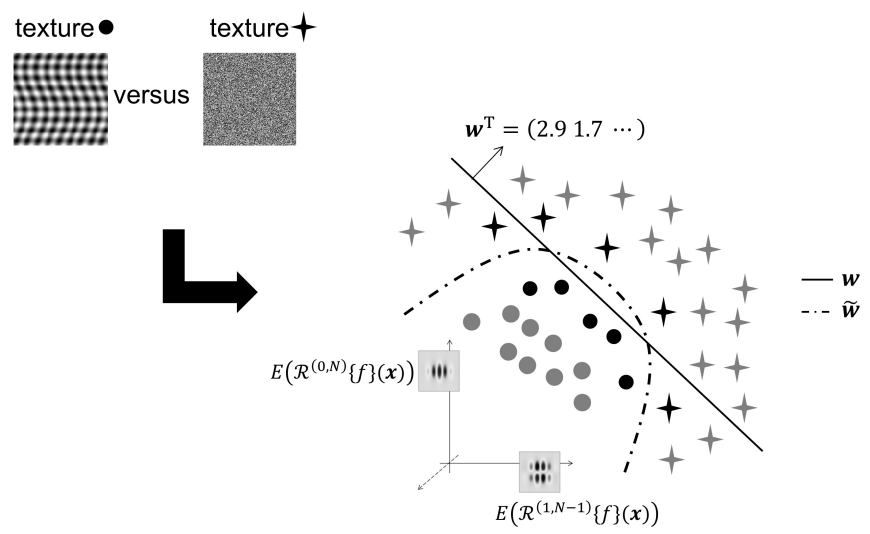

Fig. 3. Expression of the weights $w$ using the support vectors (in black) found in higher dimensional kernel space (see Eq. (9)).

by the weight that its corresponding energy level received in Eq. (9). The one-versus-all (OVA) approach is used for multiclass classification (i.e., class $c$ versus all other $\bar{c}$ ).

\section{Iterative Texture Learning and Discrimination}

The cornerstone of our approach is to locally align the learned texture signatures to maximize their response. We show that this can be done analytically and the expression of the rotated signatures remains a linear combination of the initial Riesz templates. This allows to iteratively derive texture signatures by learning a new signature $\Gamma_{c, k+1}^{N}$ in the space spanned by the maximal responses of $\Gamma_{c, k}^{N}$.

1) Steering Texture Signatures: By combining Eqs. (3) and (4), the response of a signature $\Gamma_{c}^{N}$ rotated by an arbitrary angle $\theta$ can be derived analytically as:

$$
\Gamma_{c}^{N, \theta}=\boldsymbol{w}^{\mathrm{T}} \boldsymbol{A}^{\theta} \boldsymbol{\mathcal { R }}^{N} .
$$

The expression of $\Gamma_{c}^{N, \theta}$ can still be expressed as a linear combination of the initial Riesz templates $\mathcal{R}^{N}$.

2) Iterative Signature Learning: For each iteration $k+1$, a new texture signature $\Gamma_{c, k+1}^{N}$ is learned in the feature space spanned by the maximal responses of the steered texture signature $\Gamma_{c, k}^{N}$ over $\theta$. The dominant orientation $\theta_{\mathrm{dom}}$ of $\Gamma_{c, k}^{N}$ at the position $\boldsymbol{x}_{p}$ is

$$
\theta_{\mathrm{dom}}\left(\boldsymbol{x}_{p}\right)=\underset{\theta \in[0, \pi]}{\arg \max }\left(\boldsymbol{w}_{k}^{\mathrm{T}} \boldsymbol{A}^{\theta} \mathcal{R}^{N}\{f\}\right)\left(\boldsymbol{x}_{p}\right) .
$$

A matrix $\Theta(x)$ of all angles is obtained for all positions $\boldsymbol{x}_{p}$. Riesz templates from all scales are steered together using one unique multiscale angle matrix $\Theta_{j}(\boldsymbol{x})$, which contains local angle values from scale $j$ maximizing the magnitude of $\Gamma_{c, j}^{N}$.

\section{E. Summary}

Our approach for iteratively learning the texture signature $\Gamma_{c}^{N}$ of a given class $c$ versus all others $\bar{c}$ is summarized in Fig. 4. All training examples are first represented in terms of the energies of the multiscale Riesz templates as

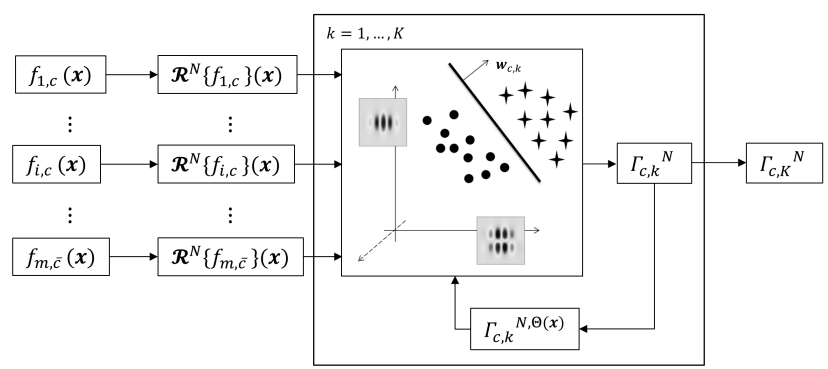

Fig. 4. Flowchart of the iterative approach to learn the texture signature $\Gamma_{c}^{N}$ of the class $c$ versus all others $\bar{c}$.

$E\left(\mathcal{R}^{(n, N-n)}\{f\}(\boldsymbol{x})\right)$. An initial texture signature is learned $\Gamma_{c, 1}^{N}$ for the class $c$ using the SVM weighting scheme described in Eq. (4) and (9). The local orientations of this initial signature are then optimized to maximize the local magnitude of $\Gamma_{c, 1}^{N}$, which reorganizes the feature space spanned by the energies of the responses of the Riesz templates. This procedure is repeated $K$ times to obtain a final rotation covariant texture signature $\Gamma_{c, K}^{N}$. As soon as $\Gamma_{c, K}^{N}$ have been learned for each class, the coefficients from all images are iteratively rotated to locally align each signature $\Gamma_{c, K}^{N}$. The coefficients are concatenated to constitute the final feature space of dimensionality $(N+1) \times J \times N_{c}$, where $N_{c}$ is the total number of classes.

\section{F. Data Sets}

We evaluated the proposed framework using both the Outex database [37] and the Brodatz collection of textures [38]. Test suites designed for extensively testing the rotation-invariant properties of the algorithms exist for both datasets. The test suites come with pre-defined training and testing sets, which allows for direct performance comparisons between approaches (i.e., identical validation methods). The cardinalities of the classes are balanced both in the training and test sets for all problems. The test suites for Outex and Brodatz are Outex_TC_00010, Outex_TC_00012 and Contrib_TC_00000 ${ }^{2}$.

1) Outex_TC_O0010 and Outex_TC_00012: Outex is a publicly available set of real textures photographed with controlled illumination conditions for the experimental evaluation of texture classification algorithms. It has recently been used by several studies on texture recognition [14], [16], [19], [21], [24]-[27], [29], [30], [33], [39], [42], [51]. It consists of 24 texture classes with pronounced directional structures. For each class, the underlying texture patterns are roughly uniform over the whole initial images of size $538 \times 746$, although gray-scale variations caused by color variations of the photographs exist. Three different color spectra were used for image capture to evaluate illumination invariance of approaches: 2300 Kelvin (K) horizon sunlight denoted as "horizon", $2856 \mathrm{~K}$ incandescent denoted as "inca", and $4000 \mathrm{~K}$ fluorescent t184 denoted as "t184". Each texture sample was captured using nine rotation angles $\left(0^{\circ}, 5^{\circ}, 10^{\circ}\right.$,

\footnotetext{
${ }^{2}$ http://www. outex.oulu.fi/, as of 20 October 2013.
} 


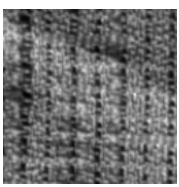

1) canvas 001

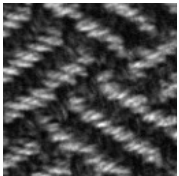

9) canvas 022

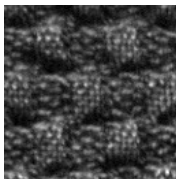

17) canvas 038

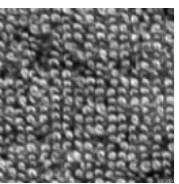

2) canvas002

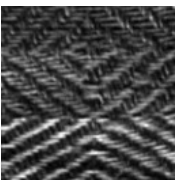

10) canvas 023

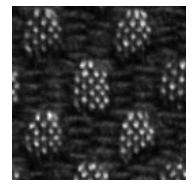

18) canvas 039

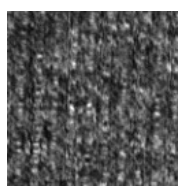

3) canvas 003

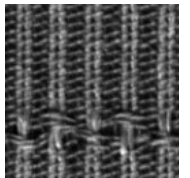

11) canvas 025

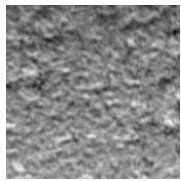

19) tile005

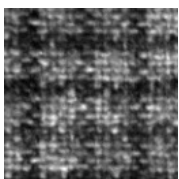

4) canvas005

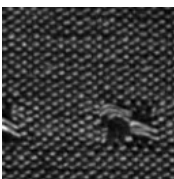

12) canvas 026

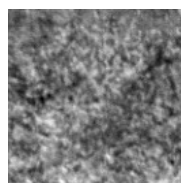

20) tile006

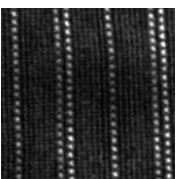

5) canvas006

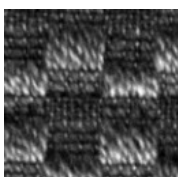

13) canvas 031

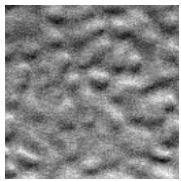

21) carpet002

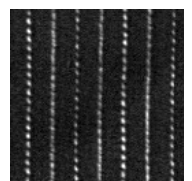

6) canvas009

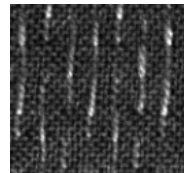

14) canvas 032

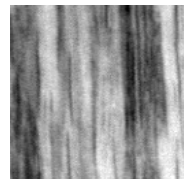

22) carpet004

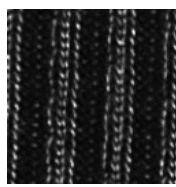

7) canvas 011

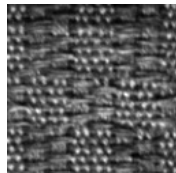

15) canvas 033

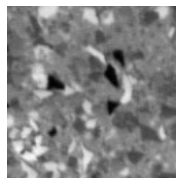

23) carpet005

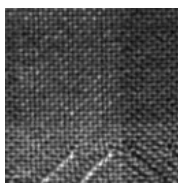

8) canvas 021

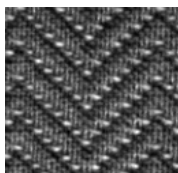

16) canvas 035

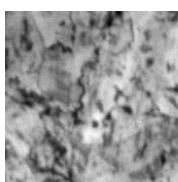

24) carpet009

Fig. 5. $128 \times 128$ blocks from the 24 texture classes of the Outex database.

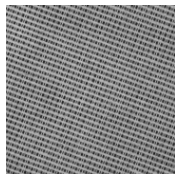

1) canvas

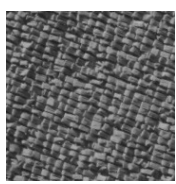

9) raffia

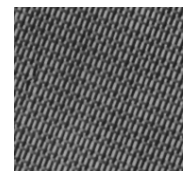

2) cloth

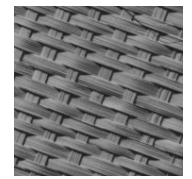

10) rattan

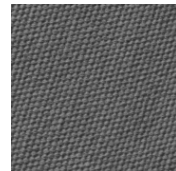

3) cotton

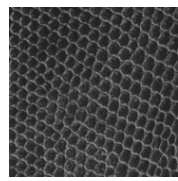

11) reptile

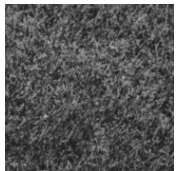

4) grass

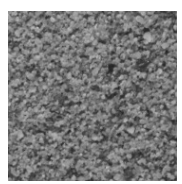

12) sand

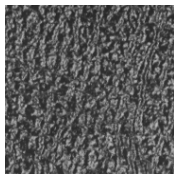

5) leather

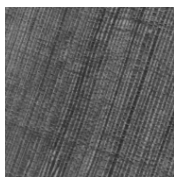

13) straw

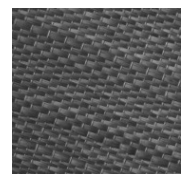

6) matting

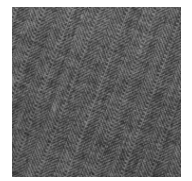

14) weave

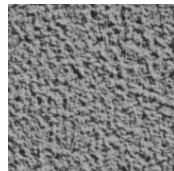

7) paper

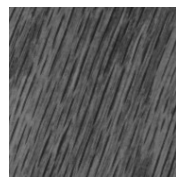

15) wood

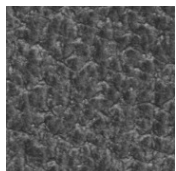

8) pigskin

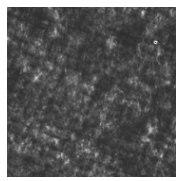

16) wool

Fig. 6. 16 Brodatz texture classes of the Contrib_TC_00000 test suite

$15^{\circ}, 30^{\circ}, 45^{\circ}, 60^{\circ}, 75^{\circ}$, and $90^{\circ}$ ) to focus on the rotationinvariant properties of the approaches. The full images were divided into $128 \times 128$ non-overlapping blocks, leading to 20 texture instances per class. Texture instances for each class are depicted in Fig. 5. The Outex_TC_00010 test suite has a total of $4320(24 \times 20 \times 9)$ image instances of illuminant "inca". The training set consists of the $480(24 \times 20)$ non-rotated images and the remaining $3840(24 \times 20 \times 8)$ images from 8 orientations are constituting the test set. Outex_TC_00012 includes two subproblems: P_000 and P_001. Both problems use the same training set as in Outex_TC_00010 (i.e., $24 \times 20$ non-rotated images of illuminant "inca"). The test sets consist of all samples captured using illuminant "tl84" for P_000 and "horizon" for P_001 and contains 4320 images.

2) Contrib_TC_00000: The Contrib_TC_00000 consists of 16 texture classes from the Brodatz album [3] depicted in Fig. 6 . For each texture class, there were eight $256 \times 256$ source images, of which the first was used for training the classifier, while the other seven images were used for testing. $180 \times 180$ images of rotated textures were created from these source images using bilinear interpolation. The training set contains $48416 \times 16$ subimages from rotation angles $0^{\circ}, 30^{\circ}, 45^{\circ}$ and $60^{\circ}$ of the first Brodatz images for each class. The total number of subimages in the training set is 7,744 . The small size of the training samples increases the difficulty of the classification task [39]. The test set is composed of $42180 \times 180$ images from rotation angles $20^{\circ}, 70^{\circ}, 90^{\circ}, 120^{\circ}, 135^{\circ}$ and $150^{\circ}$ of the other seven Brodatz images for each class. The total number of images in the test set is 672 .

\section{G. Experimental Setup}

OVA SVM models using Gaussian kernels as $K\left(\boldsymbol{x}_{i}, \boldsymbol{x}_{\boldsymbol{j}}\right)=\exp \left(\frac{-\left\|x_{i}-x_{j}\right\|^{2}}{2 \sigma_{k}^{2}}\right)$ are used both to learn texture signatures and to classify the texture instances in the final feature space obtained after $k$ iterations. A number of scales $J=6$ was used to cover the whole spectrum of the $128 \times 128$ subimages in Outex and $J=3$ for covering the spectrum of $16 \times 16$ subimages in Contrib_TC_00000. The angle matrix that maximizes the response of the texture signature at the smallest scale $\Theta_{1}(\boldsymbol{x})$ (see Eq. (11)) is used to steer Riesz templates from all scales. The dimensionality of the initial feature space is $J(N+1)$. Every texture signature $\Gamma_{c, K}^{N}$ is computed using the texture instances from the training set. The coefficients from all instances are rotated to locally align each signature $\Gamma_{c, K}^{N}$ and are concatenated to 
TABLE I

Average $A_{c}$ Obtained With the Local Orientation Maximization of $\Gamma_{c, 1}^{N}$. The Performance OF EVEN AND ODD ORDERS IS COMPARED

\begin{tabular}{c|c|c|c|c|} 
& Outex_TC_00010 & Outex_TC_00012 (P_000) & Outex_TC_00012 (P_001) & Contrib_TC_00000 \\
\hline $\bar{A}_{c}$ for $N=2,4,6,8,10$. & $98 \pm 0.7$ & $97.2 \pm 0.7$ & $98 \pm 0.4$ & $98.1 \pm 4.2$ \\
$\overline{A_{c}}$ for $N=1,3,5,7,9$. & $94.4 \pm 0.7$ & $93.6 \pm 0.6$ & $95.1 \pm 0.4$ & $89.9 \pm 1.8$ \\
$\bar{A}_{c}$ for $N=1, \ldots, 10$. & $96.2 \pm 2$ & $95.4 \pm 2$ & $96.6 \pm 1.6$ & $94 \pm 5.3$ \\
\hline
\end{tabular}

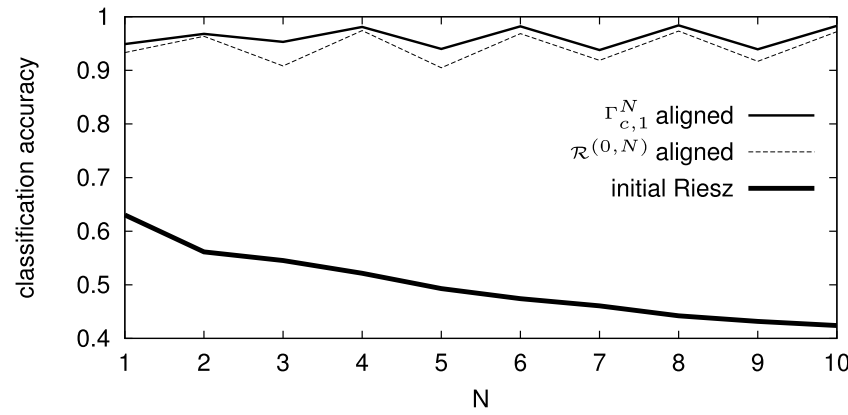

Fig. 7. Classification performance with the Outex_TC_00010 test suite. The two rotation covariant approaches are performing much better than using the initial Riesz coefficients. The local orientation maximization of $\Gamma_{c, 1}^{N}$ outperforms the local orientation of the first Riesz template $\mathcal{R}^{(0, N)}$ as proposed in [8]. A maximum $A_{c}$ of $98.4 \%$ is reached with $N=8$.

constitute the final feature space. The dimensionality of the final feature space is $J \times(N+1) \times N_{c}$. OVA SVM models are trained in this final feature space using the training instances. The remaining test instances obtained are used to evaluate the generalization performance. All data processing was performed using MATLAB R2012b (8.0.0.783) 64-bit (glnxa64), The MathWorks Inc., 2012. The computational complexity is dominated by the local orientation of $\Gamma_{c}^{N}$ in Eq. 11, which consists of finding the roots of the polynomials defined by the steering matrix $\boldsymbol{A}^{\theta}$. It is therefore NP-hard (Non-deterministic Polynomial-time hard), where the order of the polynomials is controlled by the order of the Riesz transform $N$.

\section{RESULTS}

The performance of our approach is demonstrated with the Outex and the Brodatz databases. The performance of texture classification is first investigated in Section III-A. The evolution and the convergence of the texture signatures $\Gamma_{c, k}^{N}$ through iterations $k=1, \ldots, 10$ is then studied in Section III-B for the Outex_TC_00010 test suite.

\section{A. Rotation-Covariant Texture Classification}

The rotation-covariant properties of our approach are evaluated using Outex_TC_00010, Outex_TC_00012 and Contrib_TC_00000 test suites. The classification performance of the proposed approach after the initial iteration $(k=1)$ is compared with two other approaches that are based on multiscale Riesz filterbanks. As a baseline, the classification performance using the energy of the coefficients of the initial

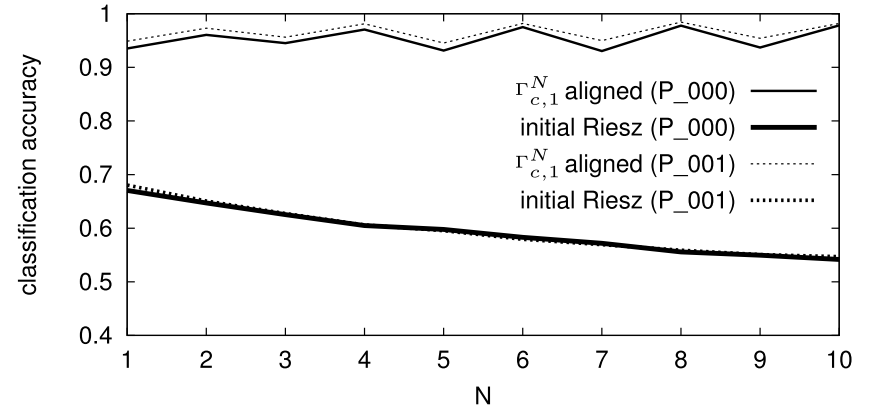

Fig. 8. Classification performance with the Outex_TC_00012 test suite for P_000 and P_001. Similarly to Outex_TC_00010, the local orientation maximization of $\Gamma_{c, 1}^{N}$ performs much better than using the initial Riesz coefficients for both problems. Maximum $A_{c}$ of $97.8 \%$ (P_000, $N=10$ ) and $98.4 \%\left(\mathrm{P} \_001, N=8\right)$ are reached, which suggests high robustness to illumination changes of the proposed approach.

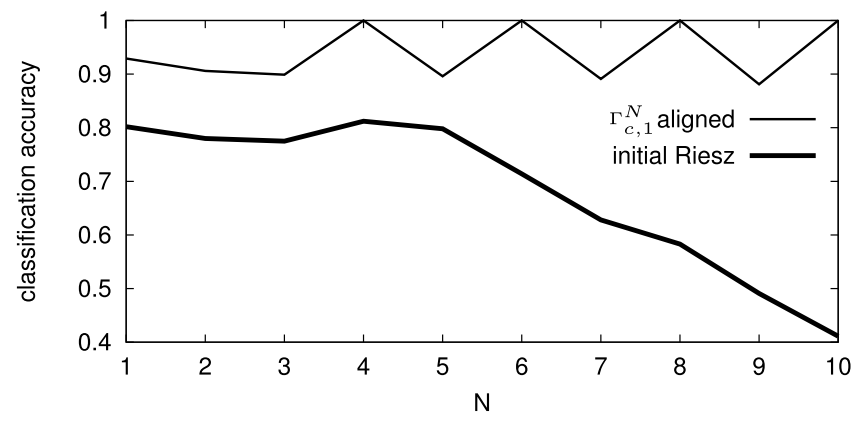

Fig. 9. Classification performance with the Contrib_TC_00000 test suite. The importance of the rotation-covariance of the operators is highlighted once more, where the local orientation maximization of $\Gamma_{c, 1}^{N}$ also performs much better than using the initial Riesz coefficients. A perfect classification $\left(A_{c}=100 \%\right)$ is reached for orders $N=4,6,8,10$.

Riesz templates was evaluated. Since the cardinality of the classes are balanced, the classification accuracy $A_{c}$ is used as a performance measure of the methods. All performances are summarized in Table I.

1) Outex_TC_00010: The classification performance for orders $N=1, \ldots, 10$ is shown in Fig. 7. The performance using the energy of the coefficients that are maximizing the response of the first Riesz template (i.e., $\mathcal{R}^{(0, N)}$ ) at the smallest scale was also evaluated as a first rotation-covariant approach [8]. An average $A_{c}$ of $96.2 \pm 2 \%$ is obtained with $N=1 \ldots 10$ and the local orientation maximization of $\Gamma_{c, 1}^{N}$. A maximum $A_{c}$ of $98.4 \%$ is reached with $N=8$.

2) Outex_TC_00012: The classification performance for orders $N=1, \ldots, 10$ is shown in Fig. 8. Average $A_{c}$ of $95.4 \pm 2 \%\left(\mathrm{P}_{-} 000\right)$ and $96.6 \pm 1.6 \%\left(\mathrm{P}_{-} 001\right)$ are obtained 


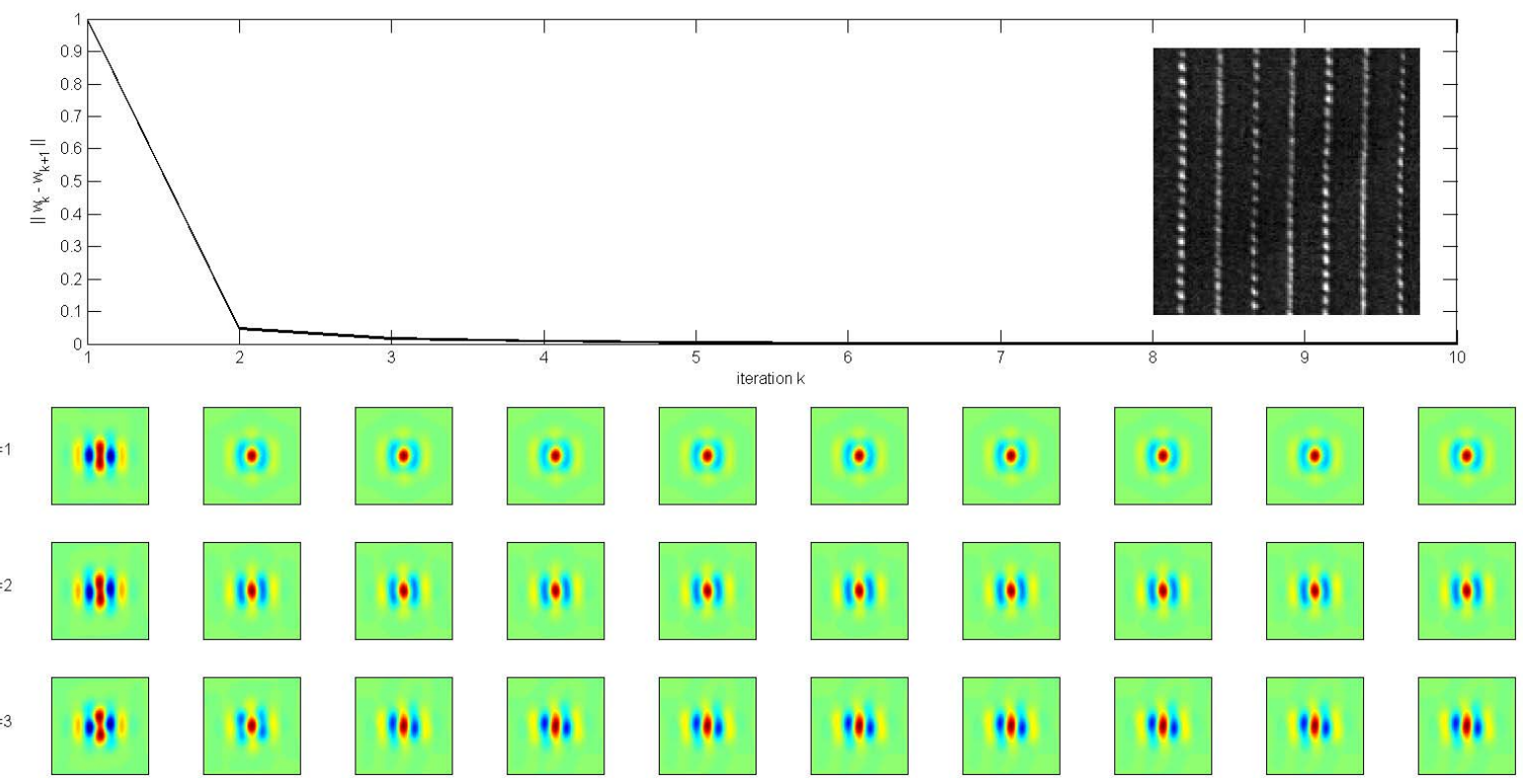

Fig. 10. Evolution and convergence of $\Gamma_{c, k}^{8}$ for class "6) canvas009" for scales $j=1,2,3$ (Outex_TC_00010). The distribution of scale-wise weights for $k=1$ and scales $j=\{1, \ldots, 6\}$ are $\{12.5 \%, 32.5 \%, 32 \%, 11.5 \%, 6.2 \%, 5.2 \%\}$. The signature is initialized as a ridge detector profile, which quickly evolves and converges to a dot detector for the smallest scale (i.e., $j=1$ ) and a ridge detector for higher significant scales (i.e., $j=2,3$ ). This is in accordance with the discriminative properties of the texture class, which is characterized by vertical lines of dots.

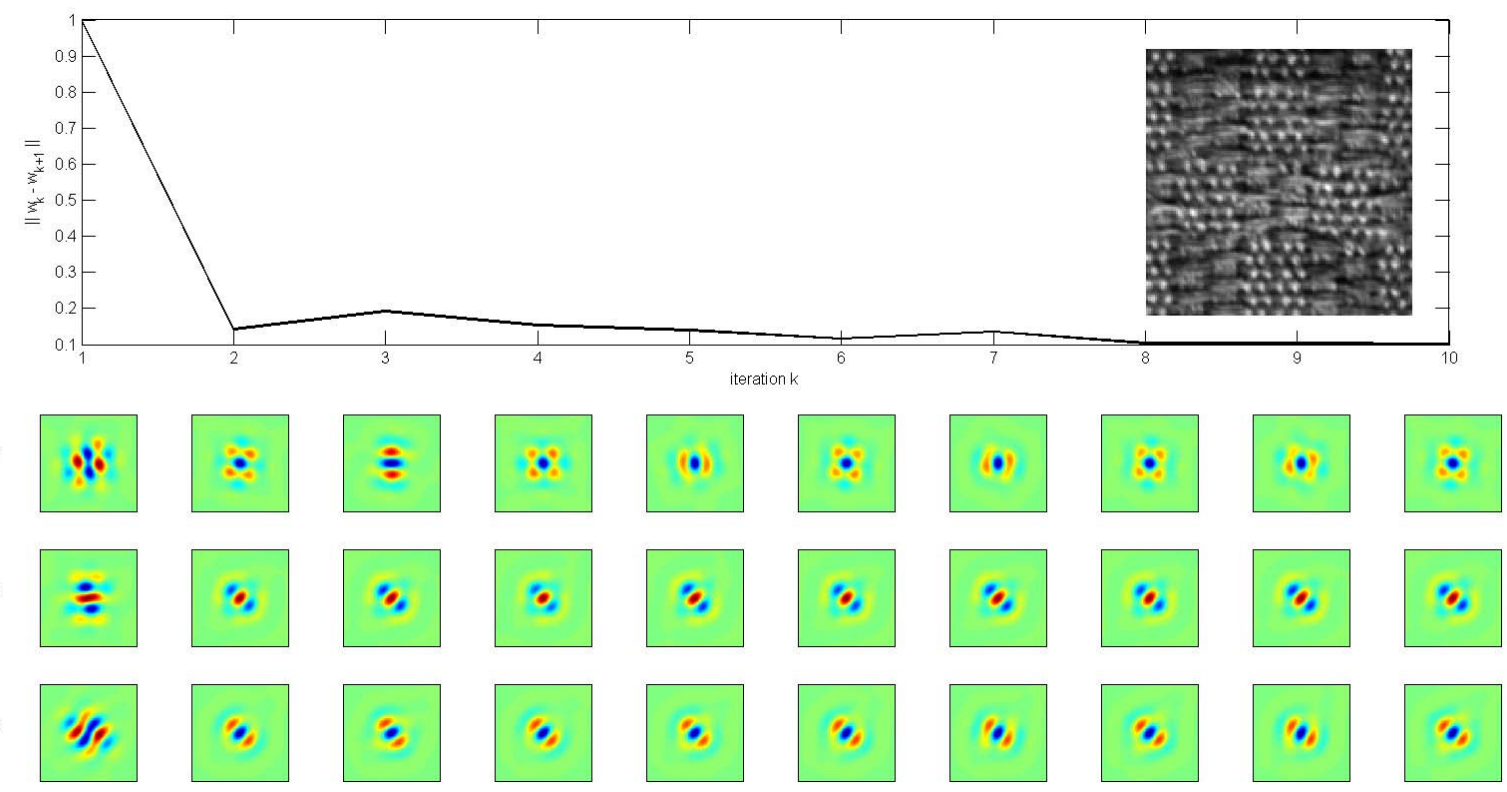

Fig. 11. Evolution and convergence of $\Gamma_{c, k}^{8}$ for class "15) canvas033" for scales $j=1,2,3$ (Outex_TC_00010). The distribution of scale-wise weights for $k=1$ and scales $j=\{1, \ldots, 6\}$ are $\{17.1 \%, 41.1 \%, 11.4 \%, 20.1 \%, 4.3 \%, 6.1 \%\}$. The associated signature oscillates from one iteration to another between a checkerboard and a ridge detector.

with $N=1 \ldots 10$ and the local orientation maximization of $\Gamma_{c, 1}^{N}$. Maximum $A_{c}$ of $97.8 \%\left(\mathrm{P}_{-} 000, N=10\right)$ and $98.4 \%$ $\left(\mathrm{P} \_001, N=8\right)$ are reached.

3) Contrib_TC_00000: The classification performance for orders $N=1, \ldots, 10$ is shown in Fig. 9. An average $A_{c}$ of $94 \pm 5.3 \%$ is obtained with $N=1 \ldots 10$ and the local orientation maximization of $\Gamma_{c, 1}^{N}$. A perfect classification of the test set $\left(A_{c}=100 \%\right)$ is reached for orders $N=4,6,8,10$.

\section{B. Convergence and Iterative Evolution of $\Gamma_{c, k}^{N}$}

The visual appearance and convergence of the texture signatures $\Gamma_{c, k}^{N}$ is investigated using the Outex_TC_00010 test suite. The assumption is made that after a given number of iterations $K, \Gamma_{c, k}^{N}$ will converge to a final template representing the essential "stitch" of the texture class. The evolutions of $\Gamma_{c, k}^{8}$ for classes "6) canvas009" and "15) canvas033" are represented in Figs. 10 and 11, respectively. The convergence is assessed 


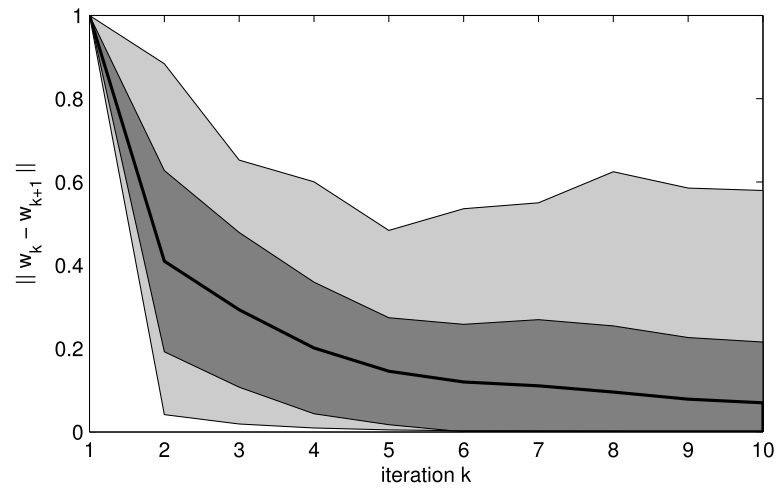

Fig. 12. Average convergence of $\Gamma_{c, k}^{8}$ for all 24 classes (Outex_TC_00010). The total range between minimum and maximum values is filled with light gray and the darker area marks the standard deviation.
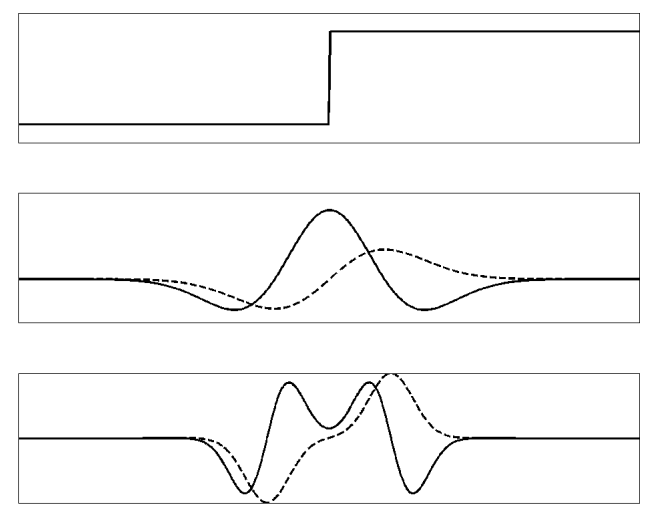

Fig. 13. Illustration of edge characterization with 1st and 2nd Gaussian derivatives in 1D. Top: Heaviside function, middle: 1st (dashed) and 2nd Gaussian derivatives and bottom: convolutions of the Gaussian derivatives with the Heaviside function. The second (i.e., even) order allows more accurate characterization of edges than first (i.e., odd) one.

as the evolution of $\frac{\left\|\boldsymbol{w}_{k}-\boldsymbol{w}_{k+1}\right\|}{\left\|\boldsymbol{w}_{1}\right\|}$. The average convergence of all classes is shown in Fig. 12.

\section{Discussions AND CONCLUSION}

We developed a texture learning framework that leverages a key property of visual pattern discrimination: the local organizations of scales and directions. Localized comprehensive characterizations of scales and directions are enabled using steerable Riesz wavelets. Class-wise templates are learned from the data: discriminative combinations of scales and directions are revealed from one-versus-all SVMs. The local orientation of the obtained templates or "signatures" is optimized to maximize their response, which is carried out analytically with linear combinations of the initial Riesz templates. The global process is iteratively repeated based on the feature space resulting from the previous iteration.

Fig. 12 shows the rapid convergence of the signatures for all classes of the Outex_TC_00010 test suite, which demonstrates that our approach is able to project texture instances into a locally rotation-covariant feature space after a small number of iterations. Both signatures and convergence behavior are stable when varying the SVM parameters (i.e., $C$ and $\sigma_{k}$ ), despite the fact that there is no guarantee of convergence to the global optimum. However, preliminary experiments using a linear kernel did not allow a systematic convergence of the signatures, which highlights the need for non-linear separation of OVA configurations. Other feature weighting schemes in Eq. (4) will be investigated in future work. The analysis of the iterative evolution of the signatures for classes "6) canvas009" and "15) canvas033" is shown in Figs. 10 and 11, respectively. A very quick convergence is observed for class 6). The signature is initialized as a ridge detector profile, which quickly evolves and converges to a dot detector for the smallest scale (i.e., $j=1$ ) and a ridge detector for higher significant scales (i.e., $j=2,3$ ). This is in accordance with the discriminative properties of the texture class, which is characterized by vertical lines of dots. The convergence is slightly slower for class 18). Initiated as a checkerboard detector, the signature evolves towards a mix of a dot and line detector, which are both present in the texture instances of class 18). Similar texture properties are found in class 15), where checkerboardlike patterns and filamentous structures coexist. It can be observed that the associated signature oscillates from one iteration to another between a checkerboard and a ridge detector and hardly converges. Future work will investigate the use of several signatures per class to allow for better characterizations of classes with several heterogeneous distinct patterns [7].

As shown in Fig. 7, we found excellent and stable overall classification performance for $N=1, \ldots, 10$ for Outex_TC_00010, Outex_TC_00012 (P_000 and P_001) and Contrib_TC_00000. The importance of rotation covariance is highlighted, where the alignment of $\Gamma_{c, k}^{N}$ achieves much better performance when compared to the initial Riesz coefficients. The performance obtained with the initial Riesz coefficients in both problems of Outex_TC_00012 is slightly higher when compared to Outex_TC_00010, because the 480 unrotated images do not require rotation-invariance of the descriptors. The importance of aligning structures (i.e., learned signatures) that correspond to the data when compared to generic templates is demonstrated, where aligning $\Gamma_{c, k}^{N}$ performs better than aligning $\mathcal{R}^{(0, N)}$ for all orders with Outex_TC_00010. This is observed even when $\mathcal{R}^{(0, N)}$ has a very strong angular selectivity with high orders of the Riesz transform. In Table I, it is observed that even orders are performing better than odd ones for all datasets, which can be explained by the fact that even orders allow more accurate characterization of edges than odd ones [49]. This is demonstrated in Fig. 13 with first and second Gaussian derivatives. The concatenation of even and odd orders (i.e., $N$ and $N-1$ ) will be investigated in future work, which will allow to model both symmetric and antisymmetric functions [55].

Even orders $N \geq 4$ enable sufficient flexibility to learn distinctive texture signatures (see Figs. 7-9). A perfect classification $\left(A_{c}=100 \%\right)$ is reached with $N=4,6,8,10$ for Contrib_TC_00000. The texture classes from the Brodatz album in Contrib_TC_00000 are more homogeneous that in Outex, where some classes clearly contain two or three visual concepts. The best performance is achieved with $N=8$ for Outex_TC_00010, which yields a confusion matrix that is 
almost diagonal. The majority of the errors are resulting from confusion between classes 3) and 14), 19) and 20), as well as between classes 22) to 24). Locally aligning signatures from large scales should reduce the confusions between 19) and 20 ), as well as 22) to 24), where the local structure is similar and the discriminative properties are occurring only at large scales. The proposed approach does not guarantee that the signatures converge to a global optimum. However, the multiscale structure (i.e., coarser scales) fosters the convergence towards a solution based on all scales, since the reorientation of the signatures is applied to all scales together. The finer scales are dragged along by coarser scales. Future work will also include the use of the steerable pyramid by Simoncelli et al. [50] as an alternative to the isotropic multiscale framework used in this work. The proposed method outperforms most filter- and wavelet-based approaches using the Outex_TC_00010 test suite [1], [26], [27], [29], [39], [42], [43], [51], where only few methods based on LBPs achieve a performance above 98\% [14], [19], [21], [24], [25], [30], [33], sometimes with manual parameter optimization. MR8, LM and S filterbanks were reported to obtain performances of $72.57 \%, 51.8 \%$ and 68.61\% when combined with SVMs in [16] in 2012 using the same database. The performance obtained for P_000 and P_001 in Outex_TC_00012 is similar to the performance obtained with Outex_TC_00010, which suggests higher illumination invariance of the proposed approach when compared to methods based on LBPs in [14], [19], [21], [30], [39]. Mother wavelet functions have zero mean, which allows encoding signal transients only and yields illumination-invariant image analysis as long as the image contrast remains unal-

$$
\begin{aligned}
& \mathcal{R}^{N} f^{\theta}(\boldsymbol{x})=\underbrace{\left(\begin{array}{c}
\mathcal{R}^{(0, N)}\left\{f^{\theta}\right\}(\boldsymbol{x}) \\
\vdots \\
\mathcal{R}^{(n, N-n)}\left\{f^{\theta}\right\}(\boldsymbol{x}) \\
\vdots \\
\mathcal{R}^{(N, 0)}\left\{f^{\theta}\right\}(\boldsymbol{x})
\end{array}\right)}_{\mathcal{R}^{N}\left\{f^{\theta}\right\}(\boldsymbol{x})}=\underbrace{\left(\begin{array}{ccccc}
A_{0,0}^{\theta} & \ldots & A_{0, l}^{\theta} & \ldots & A_{0, N}^{\theta} \\
\vdots & & \vdots & & \vdots \\
A_{n, 1}^{\theta} & \ldots & A_{n, l}^{\theta} & \ldots & A_{n, N}^{\theta} \\
\vdots & & \vdots & & \vdots \\
A_{N, 1}^{\theta} & \ldots & A_{N, l}^{\theta} & \ldots & A_{N, N}^{\theta}
\end{array}\right)}_{\boldsymbol{A}^{\theta}} \underbrace{\left(\begin{array}{c}
\mathcal{R}^{(0, N)}\{f\}(\boldsymbol{x}) \\
\vdots \\
\mathcal{R}^{(l, N-l)}\{f\}(\boldsymbol{x}) \\
\vdots \\
\mathcal{R}^{(N, 0)}\{f\}(\boldsymbol{x})
\end{array}\right)}_{\mathcal{R}^{N}\{f\}(\boldsymbol{x})} \\
& \hat{\mathcal{R}}^{(n, N-n)}=\sqrt{\frac{N}{n !(N-n) !}} \frac{\left(-j\left(\cos (\theta) \omega_{1}+\sin (\theta) \omega_{2}\right)\right)^{n}\left(-j\left(-\sin (\theta) \omega_{1}+\cos (\theta) \omega_{2}\right)\right)^{N-n}}{\|\omega\|^{N}} \\
& =\frac{1}{\|\omega\|^{N}} \sqrt{\frac{N}{n !(N-n) !}} \sum_{k_{1}=0}^{n}\left(\begin{array}{c}
n \\
k_{1}
\end{array}\right)(\cos (\theta))^{k_{1}}\left(-j \omega_{1}\right)^{k_{1}}(\sin (\theta))^{n-k_{1}}\left(-j \omega_{2}\right)^{n-k_{1}} \\
& \sum_{k_{2}=0}^{N-n}\left(\begin{array}{c}
N-n \\
k_{2}
\end{array}\right)(\cos (\theta))^{k_{2}}\left(-j \omega_{1}\right)^{k_{2}}(\sin (\theta))^{N-n-k_{2}}\left(-j \omega_{2}\right)^{N-n-k_{2}} \\
& =\frac{1}{\|\boldsymbol{\omega}\|^{N}} \sqrt{\frac{N}{n !(N-n) !}} \sum_{k_{1}=0}^{n} \sum_{k_{2}=0}^{N-n}\left(-j \omega_{1}\right)^{k_{1}+k_{2}}\left(-j \omega_{2}\right)^{N-k_{1}-k_{2}} \\
& (-1)^{k_{2}}\left(\begin{array}{c}
n \\
k_{1}
\end{array}\right)\left(\begin{array}{c}
N-n \\
k_{2}
\end{array}\right)(\cos (\theta))^{N-n-k_{2}+k_{1}}(\sin (\theta))^{n-k_{1}+k_{2}} \\
& =\sum_{k_{1}=0}^{n} \sum_{k_{2}=0}^{N-n} \underbrace{\sqrt{\frac{N}{\left(k_{1}+k_{2}\right) !\left(N-k_{1}-k_{2}\right) !} \frac{\left(-j \omega_{1}\right)^{k_{1}+k_{2}}\left(-j \omega_{2}\right)^{N-k_{1}-k_{2}}}{\|\omega\|^{N}}}}_{\hat{\mathcal{R}}^{\left(k_{1}+k_{2}, N-k_{1}-k_{2}\right)}} \\
& \sqrt{\frac{\left(k_{1}+k_{2}\right) !\left(N-k_{1}-k_{2}\right) !}{n !(N-n) !}}(-1)^{k_{2}} \frac{n !}{k_{1} !\left(n-k_{1}\right) !} \frac{(N-n) !}{k_{2} !\left(N-n-k_{2}\right) !} \\
& (\cos (\theta))^{N-n-k_{2}+k_{1}}(\sin (\theta))^{n-k_{1}+k_{2}} \text {. } \\
& \hat{\mathcal{R}}^{(n, N-n)}(\omega)=\sum_{l=0}^{N} \underbrace{\sqrt{\frac{N}{l !(N-l) !} \frac{\left(-j \omega_{1}\right)^{l}\left(-j \omega_{2}\right)^{N-l}}{\|\omega\|^{N}}}}_{\hat{\mathcal{R}}^{(l, N-l)}} \\
& \underbrace{\sum_{l_{1}=\max (0, l-N+n)}^{\min (l, n)}(-1)^{l-l_{1}} \sqrt{\frac{l !(N-l) !}{l_{1} !\left(n-l_{1}\right) !\left(l-l_{1}\right) !}}(\cos (\theta))^{N-n+2 l_{1}-l}(\sin (\theta))^{n-2 l_{1}+l}}_{A_{n, l}^{\theta}} .
\end{aligned}
$$


tered. We are currently extending the proposed framework to $3-\mathrm{D}[10]$, where the importance of learning local organizations of directions is essential because of the curse of dimensionality. The authors will make the implementation available to the community.

\section{APPENDIX A}

\section{Derivation of the Steering Matrix $\boldsymbol{A}^{\theta}$}

Let us consider the matrix form of Eq. (3) in Eq. (12), shown at the bottom of the previous page. To derive the explicit form of the terms $A_{n, l}^{\theta}$, let us consider the Riesz transform of a rotated image $f^{\theta}(\boldsymbol{x})$ at the origin $\mathbf{0}$. In the Fourier domain, the rotated image is $\hat{f}^{\theta}(\boldsymbol{\omega})=\hat{f}\left(\boldsymbol{\omega}^{\prime}\right)$, where $\omega_{1}^{\prime}=\cos (\theta) \omega_{1}+$ $\sin (\theta) \omega_{2}$ and $\omega_{2}^{\prime}=-\sin (\theta) \omega_{1}+\cos (\theta) \omega_{2}$. The Riesz transform of a rotated image is developed in Eq. (13), shown at the bottom of the previous page. Using the change of variables $k_{1}+k_{2}=l$ in Eq. (13), the elements $n, l$ of $\boldsymbol{A}^{\theta}$ are developed in Eq. (14), shown at the bottom of the previous page.

\section{REFERENCES}

[1] Y. Ben Salem and S. Nasri, "Rotation invariant texture classification using support vector machines," in Proc. IEEE Int. Conf. Commun., Comput. Control Appl., Mar. 2011, pp. 1-6.

[2] C. Blakemore and F. W. Campbell, "On the existence of neurones in the human visual system selectively sensitive to the orientation and size of retinal images," J. Physiol., vol. 203, no. 1, pp. 237-260, 1969

[3] P. Brodatz, Textures: A Photographic Album for Artists and Designers. New York, NY, USA: Dover, 1966

[4] E. J. Candès and D. L. Donoho, "Curvelets-A surprisingly effective nonadaptive representation for objects with edges," in Curves and Surface Fitting, Nashville, TN, USA: Vanderbilt Univ. Press, 2000, pp. $105-120$.

[5] F. H. C. Crick, D. C. Marr, and T. Poggio, "An information processing approach to understanding the visual cortex," Artificial Intelligence Laboratory, Massachusetts Institute of Technology, Cambridge, MA USA, Tech. Rep., 1980

[6] O. Cula and K. Dana, "3D texture recognition using bidirectional feature histograms," Int. J. Comput. Vis., vol. 59, no. 1, pp. 33-60, 2004

[7] A. Depeursinge, A. Foncubierta-Rodríguez, H. Müller, and D. Van De Ville, "Rotation-covariant visual concept detection using steerable Riesz wavelets and bags of visual words," Proc. SPIE, vol. 8858, pp. 885816-1-885816-11, Sep. 2013.

[8] A. Depeursinge, A. Foncubierta-Rodríguez, D. Van De Ville, and H. Müller, "Lung texture classification using locally-oriented Riesz components," in Medical Image Computing and Computer Assisted Intervention (Lecture Notes in Computer Science) vol. 6893. Berlin/Heidelberg, Germany: Springer-Verlag, 2011, pp. 231-238.

[9] A. Depeursinge, A. Foncubierta-Rodríguez, D. Van De Ville, and H. Müller, "Multiscale lung texture signature learning using the Riesz transform," in Medical Image Computing and Computer Assisted Intervention (Lecture Notes in Computer Science) vol. 7512. Berlin/Heidelberg, Germany: Springer-Verlag, 2012, pp. 517-552.

[10] A. Depeursinge, A. Foncubierta-Rodríguez, A. Vargas, D. Van De Ville, A. Platon, P.-A. Poletti, et al., "Rotation-covariant texture analysis of 4D dual-energy CT as an indicator of local pulmonary perfusion," in Proc. IEEE 10th ISBI, Apr. 2013, pp. 149-152.

[11] M. N. Do and M. Vetterli, "Contourlets: A directional multiresolution image representation," in Proc. ICIP, vol. 1. 2002, pp. 357-360.

[12] M. N. Do and M. Vetterli, "Rotation invariant texture characterization and retrieval using steerable wavelet-domain hidden Markov models," IEEE Trans. Multimedia, vol. 4, no. 4, pp. 517-527, Dec. 2002.

[13] D. L. Donoho, "Ridge functions and orthonormal ridgelets," J. Approx. Theory, vol. 111, no. 2, pp. 143-179, 2001.

[14] N. Doshi and G. Schaefer, "A comparative analysis of local binary pattern texture classification," in Proc. IEEE VCIP, Nov. 2012, pp. 1-6.

[15] W. Freeman and E. Adelson, "The design and use of steerable filters," IEEE Trans. Pattern Anal. Mach. Intell., vol. 13, no. 9, pp. 891-906, Sep. 1991

[16] O. Ghita, D. Ilea, A. Fernandez, and P. Whelan, "Local binary patterns versus signal processing texture analysis: A study from a performance evaluation perspective," Sensor Rev., vol. 32, no. 2, pp. 149-162, 2012.

[17] G. Gonzalez, F. Fleuret, and P. Fua, "Learning rotational features for filament detection," in Proc. IEEE Conf. CVPR, Jun. 2009, pp. $1582-1589$.
[18] H. Greenspan, S. Belongie, R. Goodman, P. Perona, S. Rakshit, and C. H. Anderson, "Overcomplete steerable pyramid filters and rotation invariance," in Proc. IEEE Comput. Soc. Conf. CVPR, Jun. 1994, pp. $222-228$.

[19] Z. Guo, Q. Li, J. You, D. Zhang, and W. Liu, "Local directional derivative pattern for rotation invariant texture classification," Neural Comput. Appl., vol. 21, no. 8, pp. 1893-1904, 2012.

[20] Z. Guo, Q. Li, L. Zhang, J. You, D. Zhang, and W. Liu, "Is local dominant orientation necessary for the classification of rotation invariant texture," Neurocomputing, vol. 116, pp. 182-191, Sep. 2013.

[21] Z. Guo, L. Zhang, and D. Zhang, "A completed modeling of local binary pattern operator for texture classification," IEEE Trans. Image Process., vol. 19 , no. 6 , pp. 1657-1663, Jun. 2010.

[22] I. Guyon, J. Weston, S. Barnhill, and V. Vapnik, "Gene selection for cancer classification using support vector machines," Mach. Learn., vol. 46, nos. 1-3, pp. 389-422, 2002.

[23] R. M. Haralick, K. Shanmugam, and I. Dinstein, "Textural features for image classification," IEEE Trans. Syst., Man Cybern., vol. 3, no. 6, pp. 610-621, Nov. 1973

[24] Y. He, N. Sang, and C. Gao, "Pyramid-based multi-structure local binary pattern for texture classification," in Computer Vision (Lecture Notes in Computer Science), vol. 6494. Berlin Heidelberg, Germany: SpringerVerlag, 2011, pp. 133-144.

[25] Y. He, N. Sang, and R. Huang, "Local binary pattern histogram based texton learning for texture classification," in Proc. 18th IEEE ICIP, Sep. 2011, pp. 841-844.

[26] K. Jafari-Khouzani and H. Soltanian-Zadeh, "Radon transform orientation estimation for rotation invariant texture analysis," IEEE Trans. Pattern Anal. Mach. Intell., vol. 27, no. 6, pp. 1004-1008, Jun. 2005.

[27] P. Janney and Z. Yu, "Invariant features of local textures-A rotation invariant local texture descriptor," in Proc. IEEE Conf. Comput. Vis. Pattern Recognit., 2007, pp. 1-7.

[28] B. Julesz, "Visual pattern discrimination," IRE Trans. Inf. Theory, vol. 8, no. 2, pp. 84-92, 1962.

[29] U. Kandaswamy, S. A. Schuckers, and D. Adjeroh, "Comparison of texture analysis schemes under nonideal conditions," IEEE Trans. Image Process., vol. 20, no. 8, pp. 2260-2275, Aug. 2011.

[30] F. Khellah, "Texture classification using dominant neighborhood structure," IEEE Trans. Image Process., vol. 20, no. 11, pp. 3270-3279, Nov. 2011.

[31] S. Lazebnik, C. Schmid, and J. Ponce, "A sparse texture representation using local affine regions," IEEE Trans. Pattern Anal. Mach. Intell., vol. 27 , no. 8, pp. 1265-1278, Aug. 2005.

[32] T. Leung and J. Malik, "Representing and recognizing the visual appearance of materials using three-dimensional textons," Int. J. Comput. Vis., vol. 43, no. 1, pp. 29-44, 2001.

[33] L. Liu, L. Zhao, Y. Long, G. Kuang, and P. Fieguth, "Extended local binary patterns for texture classification," Image Vis. Comput., vol. 30, no. 2, pp. 86-99, 2012.

[34] D. G. Lowe, "Distinctive image features from scale-invariant keypoints," Int. J. Comput. Vis., vol. 60, no. 2, pp. 91-110, 2004.

[35] S. G. Mallat, "A theory for multiresolution signal decomposition: The wavelet representation," IEEE Trans. Pattern Anal. Mach. Intell., vol. 11, no. 7, pp. 674-693, Jul. 1989.

[36] F. G. Meyer and R. R. Coifman, "Brushlets: A tool for directional image analysis and image compression," Appl. Comput. Harmon. Anal., vol. 4, no. 2, pp. 147-187, 1997

[37] T. Ojala, T. Mäenpää, M. Pietikäinen, J. Viertola, J. Kyllönen, and S. Huovinen, "Outex-New framework for empirical evaluation of texture analysis algorithms," in Proc. 16th ICPR, vol. 1. 2002, pp. 701-706.

[38] T. Ojala, M. Pietikäinen, and T. Mäenpää, "Gray scale and rotation invariant texture classification with local binary patterns," in Computer Vision (Lecture Notes in Computer Science), vol. 1842. Berlin Heidelberg, Germany: Springer-Verlag, 2000, pp. 404-420.

[39] T. Ojala, M. Pietikäinen, and T. Mäenpää, "Multiresolution gray-scale and rotation invariant texture classification with local binary patterns," IEEE Trans. Pattern Anal. Mach. Intell., vol. 24, no. 7, pp. 971-987, Jul. 2002.

[40] P. Perona, "Deformable kernels for early vision," IEEE Trans. Pattern Anal. Mach. Intell., vol. 17, no. 5, pp. 488-499, May 1995.

[41] D. D. Y. Po and M. N. Do, "Directional multiscale modeling of images using the contourlet transform," IEEE Trans. Image Process., vol. 15, no. 6 , pp. $1610-1620$, Jun. 2006

[42] R. Porter and N. Canagarajah, "Robust rotation-invariant texture classification: Wavelet, Gabor filter and GMRF based schemes," IEE Proc. Vis., Image Signal Process., vol. 144, no. 3, pp. 180-188, Jun. 1997.

[43] X. Qian, X.-S. Hua, P. Chen, and L. Ke, "PLBP: An effective local binary patterns texture descriptor with pyramid representation," Pattern Recognit., vol. 44, nos. 10-11, pp. 2502-2515, 2011. 
[44] G. Quellec, M. Lamard, G. Cazuguel, B. Cochener, and C. Roux, "Fast wavelet-based image characterization for highly adaptive image retrieval," IEEE Trans. Image Process., vol. 21, no. 4, pp. 1613-1623, Apr. 2012.

[45] T. Randen and J. H. Husoy, "Filtering for texture classification: A comparative study," IEEE Trans. Pattern Anal. Mach. Intell., vol. 21, no. 4, pp. 291-310, Apr. 1999.

[46] R. Rigamonti and V. Lepetit, "Accurate and efficient linear structure segmentation by leveraging ad hoc features with learned filters," in Medical Image Computing and Computer-Assisted Intervention (Lecture Notes in Computer Science), vol. 7510, N. Ayache, H. Delingette, P. Golland, and K. Mori, Eds. Berlin Heidelberg, Germany: SpringerVerlag, 2012, pp. 189-197.

[47] C. Schmid, "Constructing models for content-based image retrieval," in Proc. IEEE Comput. Soc. Conf. CVPR, vol. 2. Dec. 2001, pp. 39-45.

[48] M. Schneider, S. Hirsch, G. Székely, B. Weber, and B. H. Menze, "Oblique random forests for 3-D vessel detection using steerable filters and orthogonal subspace filtering," in Proc. MICCAI Workshop Med. Comput. Vis., 2013, pp. 142-154.

[49] E. Simoncelli and H. Farid, "Steerable wedge filters for local orientation analysis," IEEE Trans. Image Process., vol. 5, no. 9, pp. 1377-1382, Sep. 1996

[50] E. Simoncelli and W. Freeman, "The steerable pyramid: A flexible architecture for multi-scale derivative computation," in Proc. Int. Conf. Image Process., vol. 3. 1995, pp. 444-447.

[51] P. Southam and R. Harvey, "Towards texture classification in real scenes," in Proc. Brit. Mach. Vis. Conf., 2005, pp. 240-250.

[52] E. M. Stein and G. Weiss, Introduction to Fourier Analysis on Euclidean Spaces. Princeton, NJ, USA: Princeton Univ. Press, 1971.

[53] H. Tamura, S. Mori, and T. Yamawaki, "Textural features corresponding to visual perception," IEEE Trans. Syst., Man Cybern., vol. 8, no. 6, pp. 460-473, Jun. 1978.

[54] G. Tzagkarakis, B. Beferull-Lozano, and P. Tsakalides, "Rotationinvariant texture retrieval with gaussianized steerable pyramids," IEEE Trans. Image Process., vol. 15, no. 9, pp. 2702-2718, Sep. 2006.

[55] M. Unser and N. Chenouard, "A unifying parametric framework for 2D steerable wavelet transforms," SIAM J. Imag. Sci., vol. 6, no. 1, pp. 102-135, 2013.

[56] M. Unser and D. Van De Ville, "Wavelet steerability and the higherorder Riesz transform," IEEE Trans. Image Process., vol. 19, no. 3, pp. 636-652, Mar. 2010.

[57] M. Unser, D. Van De Ville, and N. Chenouard, "Steerable pyramids and tight wavelet frames in $L_{2}\left(\mathbb{R}^{d}\right)$," IEEE Trans. Image Process., vol. 20 , no. 10 , pp. $2705-2721$, Oct. 2011.

[58] D. Van De Ville, T. Blu, and M. Unser, "Isotropic polyharmonic B-splines: Scaling functions and wavelets," IEEE Trans. Image Process., vol. 14, no. 11, pp. 1798-1813, Nov. 2005.

[59] V. Vapnik, The Nature of Statistical Learning Theory. New York, NY, USA: Springer-Verlag, 1995.

[60] Y. Xu, X. Yang, H. Ling, and H. Ji, "A new texture descriptor using multifractal analysis in multi-orientation wavelet pyramid," in Proc. IEEE Comput. Soc. Conf. Comput. Vis. Pattern Recognit., Jun. 2010, pp. $161-168$.

[61] J. Zhang, M. Marszalek, S. Lazebnik, and C. Schmid, "Local features and kernels for classification of texture and object categories: A comprehensive study," Int. J. Comput. Vis., vol. 73, no. 2, pp. 213-238, 2007.

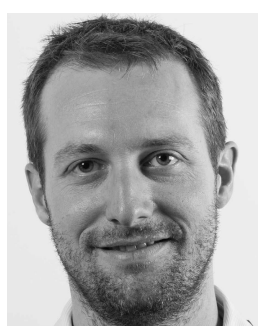

Adrien Depeursinge received the B.Sc. and M.Sc. degrees in electrical engineering from the Swiss Federal Institute of Technology, Lausanne, Switzerland, in 2003 and 2005, respectively, with a specialization in signal and image processing. From 2006 to 2010, he performed his Ph.D. thesis on medical image analysis with a focus on texture analysis and content-based image retrieval at the University Hospitals of Geneva (HUG). He then spent two years as a Post-Doctoral Fellow at the University of Applied Sciences Western Switzerland, Sierre (HES-SO), Switzerland and the HUG. He is currently with the Department of Radiology, School of Medicine, Stanford University. Dr. Depeursinge was a recipient of the 2011 German Association for Medical Informatics, Biometry and Epidemiology Award in medical informatics for his Ph.D. thesis. His research interests include (1) N-dimensional texture analysis with control of scales and orientations, (2) automated detection of semantic visual concepts (e.g. RadLex terms), and (3) clinical workflows of image-based computer-aided diagnosis systems.

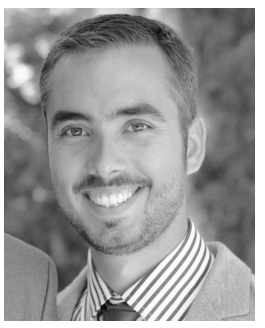

Antonio Foncubierta-Rodriguez received the M.Eng. degree in telecommunication engineering from the University of Seville, Spain, in 2009. Since 2007, he has been a part-time Researcher with the Department of Communications and Signal Processing, University of Seville. His research was related to video compression and transmission over mobile networks, leading to a master's thesis. Since 2008, he worked on a project on medical image retrieval for the University Hospitals Virgen del Rocío in Seville.

Currently, as a Ph.D. Student at the University of Geneva, he is a Research Assistant at the University of Applied Sciences Western Switzerland in Sierre, where he works on several swiss national and EU projects.

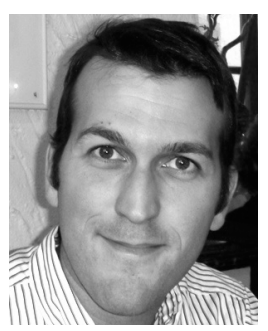

Dimitri Van de Ville (M'02-SM'12) received the M.S. degree in engineering and computer sciences and the Ph.D. degree from Ghent University, Belgium, in 1998, and 2002, respectively. After a postdoctoral stay (2002-2005) at the Biomedical Imaging Group of Prof. M. Unser at the École Polytechnique Fédérale de Lausanne (EPFL), Switzerland, he became responsible for the Signal Processing Unit, University Hospital of Geneva, Switzerland, as part of the Centre d'Imagerie Biomédicale. In 2009, he was a recipient of the Swiss National Science Foundation professorship and currently holds a joint position at the University of Geneva, Switzerland, and the EPFL. His research interests include wavelets, sparsity, pattern recognition, and their applications in biomedical imaging, such as functional magnetic resonance imaging. He received the Pfizer Research Award 2012 in the category Neurosciences for his work on scalefree dynamics of EEG microstates.

Dr. Van de Ville served as an Associate Editor for the IEEE TRANS ACTIONS ON IMAGE PROCESSING from 2006 to 2009 and the IEEE SignAL PROCESSING LETTERS from 2004 to 2006 . He is the Chair of the Bio Imaging and Signal Processing TC of the IEEE Signal Processing Society (2012-2013). Since 2003, he has been an Editor and Webmaster of The Wavelet Digest. He is Co-Chair of the biennial Wavelets \& Sparsity series conferences, together with V. Goyal and M. Papadakis.

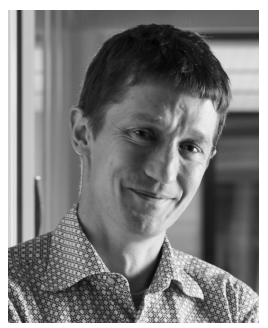

Henning Müller received the master's degree in medical informatics from Heidelberg University, Germany, in 1997, and the Ph.D. degree in multimedia information retrieval from Geneva University, Switzerland, in 2002

During this time, he also worked for Daimler Benz research and technology North America, Portland, OR, USA, and at Monash University, Melbourne, Australia. After his Ph.D., he has worked in medical informatics at the University and University hospitals of Geneva, Switzerland, where he finished his habilitation in 2008. Since 2007, he has been a Professor of computer science at the University of Applied Sciences Western Switzerland, Sierre, Switzerland.

Henning has lead the ImageCLEF benchmark on multilingual and multimodal information retrieval for ten years. He has published over 400 scientific articles on visual information analysis and retrieval. He has participated in several EU projects and has initiated several national projects. He is currently coordinator of the Khresmoi project. 